\title{
Female sexual dysfunction in patients with spinal cord injury: a study from Iran
}

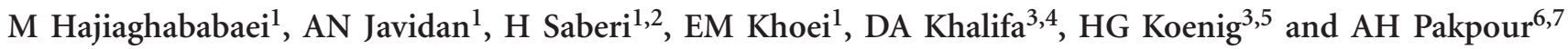

Objectives: We assess the prevalence of sexual dysfunction in patients with spinal cord injury (SCI), compare sexual function and sexual distress between female patients with $\mathrm{SCl}$ and gender-matched healthy controls, and address risk factors associated with sexual dysfunction among Iranian female patients with SCl.

Setting: Brain and Spinal Cord Injury Research Center, Neuroscience Institute, Tehran University of Medical Sciences, Tehran, Iran. Methods: Eligible Iranian female patients with SCl were included in this cross-sectional study. They were asked to provide sociodemographic information, and complete the Female Sexual Function Index, Hospital Anxiety and Depression Scale and Female Sexual Distress Scale-Revised questionnaire.

Results: Of the 105 patients participated in this study, the average age was 41.0 (s.d. $=10.1$ ) years. Women with SCl reported significantly higher levels of sexual dysfunction compared with normal controls. Approximately, $88 \%$ of $\mathrm{SCl}$ patients reported at least one type of sexual dysfunction, whereas only $37 \%$ of healthy controls reported sexual dysfunction. Lack of vaginal lubrication was reported more frequent in SCI patients compared with controls. Women with SCI reported a significantly higher level of sexual distress compared with healthy women. Sexual dysfunction was observed to be significantly higher in older patients, those with less education, patients with complete lesions, those with sexual distress and patients who were anxious and depressed.

Conclusion: Sexual dysfunction is highly prevalent among Iranian women with SCl. Sexual dysfunction is associated with age, education, symptoms of depression and anxiety and level of injury. Sexual counseling during the rehabilitation period may help to prevent sexual dysfunction following $\mathrm{SCl}$.

Spinal Cord (2014) 52, 646-649; doi:10.1038/sc.2014.99; published online 17 June 2014

\section{INTRODUCTION}

Spinal cord injury (SCI) is a common cause of severe disability and death. The annual incidence of SCI is up to 40 cases per million in the United States. ${ }^{1}$ In Iran, as a developing country, SCI relatively common and estimated 4.4 per $100000 .^{2}$ Health care and life expenditures due to SCI are about $\$ 200000$ during the first 2 years after injury. ${ }^{3}$ Patients with SCI suffer from impaired emotional, physical and social functions, lower rates of school enrollment and higher mortality rates. ${ }^{2}$ In addition, patents with SCI are more likely to suffer from impaired bladder and bowel control, fatigue and musculoskeletal pain. ${ }^{1}$ Efforts are now directed to help patients with SCI to live productive lives and experience a normal life expectancy. However, studies have found that patients with SCI suffer from sexual dysfunction and this is true for both sexes depending on the level of injury. ${ }^{4-6}$ Owing to a high prevalence of SCI in men, numerous studies have focused on the sexuality of men with SCI. Less attention, however, has been paid to problems with sexual function in women with SCI. Women are more prone to depression and psychological disorders after injury compared with men. ${ }^{4}$ Moreover, studies have found that women with SCI frequently report loss of vaginal lubrication and problems reaching orgasm. ${ }^{4-8}$
Female sexual dysfunction (FSD) can often interfere with quality of life. ${ }^{9}$ Studies have found that women with SCI believe that they should have a passive role during sexual intercourse and this adversely affects their sexual functioning. ${ }^{7}$ In a previous study that explored sexual dysfunction in females with $\mathrm{SCI},{ }^{8}$ Lombardi and collogues investigated FSD using the Female Sexual Function Index (FSFI). They found that $59 \%$ reported at least one sexual dysfunction. ${ }^{8}$ Most studies of sexual dysfunction in women with SCI involved small samples and did not compare them to women without SCI.

The aims of this study were to assess the prevalence of FSD and determine risk factors associated with FSD among women with SCI.

\section{MATERIALS AND METHODS}

\section{Patients and setting}

This cross-sectional study was conducted between January 2012 and August 2013. The study sample consisted of female patients with SCI who had been referred to the Brain and Spinal Cord Injury Repair Research Center in Tehran, Iran. Brain and Spinal Cord Injury Repair Research Center is a referral center for patients with SCI from all over the country. The inclusion criterion were age 18 years older, married or sexually active within the past 6 months, and having a SCI at any neurological level for longer than 6 months. Patients with

${ }^{1}$ Brain and Spinal Cord Injury Research Center, Neuroscience Institute, Tehran University of Medical Sciences, Tehran, Iran; ${ }^{2}$ Department of Neurosurgery Center, Imam Khomeini Hospital, Tehran University of Medical Sciences, Tehran, Iran; ${ }^{3}$ Department of Medicine, King Abdulaziz University, Jeddah, Saudi Arabia; ${ }^{4}$ Ain Shams University, Cairo, Egypt; ${ }^{5}$ Department of Medicine and Psychiatry, Duke University Medical Center, Durham, NC, USA; ${ }^{6}$ Social Determinants of Health Research Center, Qazvin University of Medical Sciences, Qazvin, Iran and ${ }^{7}$ Department of Public Health, Qazvin University of Medical Sciences, Qazvin, Iran

Correspondence: Dr AH Pakpour, Department of Public Health, Qazvin University of Medical Sciences, Shahid Bahounar BLV, Qazvin 3419759811, Iran.

E-mail: Pakpour_Amir@yahoo.com or apakpour@qums.ac.ir

Received 25 February 2014; revised 10 April 2014; accepted 7 May 2014; published online 17 June 2014 
major medical diseases, including diabetes mellitus, cardiovascular diseases, hypertension, rheumatic diseases, kidney disease, severe mental and psychiatric disorders, substance abuse or pregnant, were excluded from the study. Also excluded were patients who suffered from cognitive impairment that was assessed by the Mini-Mental State Exam. Patients were excluded from the study if they had Mini-Mental State Exam $<27$. After checking their eligibility criteria, all potential participants were informed about aims of the study and asked to read and sign an informed consent form. The study was approved by the Institutional Review Board of Qazvin University of Medical Sciences.

\section{Measures}

Sociodemographic questions included age, educational status, employment status, living situation and whether or not the participant had children. Also collected from the medical information were information about the duration of SCI in months, level of lesion and the reason for the SCI. The neurological status of the patients (that is, motor and sensory levels impaired by the SCI) was evaluated using the American Spinal Injury Association (ASIA) guidelines. ${ }^{10}$

\section{FSFI}

The 19-item FSFI is a multidimensional tool to assess females' sexuality and has been used in both clinical and community populations. The measure is composed of six subscales including sexual desire (two items), arousal (four items), lubrication (four items), orgasm (three items), satisfaction (three items) and pain (three items). All subscale scores ranged from 0 to 5 with higher scores indicating better sexual functioning. Subscale scores are summed to obtain a total score for the FSFI. Scores of 27 or lower have been identified as the cutoff for sexual dysfunction. ${ }^{11}$ The Iranian version of the FSFI has been found to be a valid and highly reliable tool for detecting sexual dysfunction among women. ${ }^{9}$

\section{Hospital anxiety and depression scale (HADS)}

The HADS is a self-administered measure of anxiety and depression that consists of 14 questions divided into two subscale (7 items for anxiety and 7 items for depression). All items are rated on a four-point response scale ranging from 0 (absence of symptoms) to 3 (severe symptoms). Both anxiety and depression scales range from 0 to 21 with higher scorers indicating higher symptoms of distress. The recommended cutoff scores for both anxiety and depression are: 0-7 (non-case), 8-10 (possible case) and 11-21 (cases). The HADS has been translated into Persian (Farsi), and its psychometric properties have been established in Iranian populations. ${ }^{12}$

\section{Female sexual distress scale-revised (FSDS-R)}

The FSDS-R is a 13-item questionnaire that assesses different aspects of sexual distress in women. All items are rated on a Likert-type-scale ranging from 0 (never) to 4 (always) with higher scores indicating greater sexual distress. Items are summed to create a total score for the FSDS-R. A score of 11 or higher is considered the cutoff for women with sexual distress. The Iranian version of the FSDS-R has acceptable psychometric properties when used in Iranian women. ${ }^{13}$

\section{Statistical analysis}

A control sample of 1123 Iranian women without SCI was taken from a previous study. ${ }^{13}$ Women in the control group did not have any systematic diseases that might interfere with sexual functioning. The control group was matched for age, educational status and having children to women with SCI. Descriptive statistics were used to summarize the characteristics of the sample, including scores on the FSFI subscales, FSDS-R and HADS. Symptoms of depression, anxiety, sexual distress and dysfunction were compared between cases and controls using the $\chi^{2}$ test or Student's $t$-test. Benjamini and Hochberg adjustments in the level of statistical significance were made to control for multiple comparisons. ${ }^{14}$

A logistic regression model was used to identify correlates of FSD (defined as an FSFI total score $\leqslant 27)$ controlling for potential confounders. In this model, FSD status (FSD $=1$ and healthy $=2$ ) was the dependent variable, whereas age, living situation, educational status, work status, ASIA grade, FSDS-R score, depression and anxiety were entered as independent variables. A forward selection method was used in the analysis, entering all variables that were significant in bivariate analyses at $P<0.10$ into the model at one time. Multicollinearity was checked by calculating the tolerance collinearity statistics and the variance inflation factor, where a tolerance less than 0.2 and variance inflation factor $>5$ indicate problems. ${ }^{15}$ All statistical analyses were done using SPSS, version 16 (SPSS, Chicago, IL, USA).

\section{RESULTS}

Of 118 eligible patients with SCI, 13 (11\%) declined to participate because of discomfort over answering questions about sexual functioning, which reflects the highly personal and private nature of sexual activity in Iran. Average age was 41.0 (10.1) years. Education level was relatively low, with only $36.2 \%$ educated at the secondary school level. A majority were from urban areas (74.3\%). Most (80.0\%) had acquired their SCI from motor vehicle accidents. The ASIA grade was A in $57.1 \%, \mathrm{~B}$ in $14.3 \%, \mathrm{C}$ in $9.5 \%, \mathrm{D}$ in $14.3 \%$ and $\mathrm{E}$ in $4.8 \%$. For about half $(43 \%)$, the site of injury was at the thoracic

\section{Table 1 Sociodemographic and clinical characteristics of the study population}

Patients with $\mathrm{SCl}(\mathrm{n}=105)$

Age, years
Living situation
Urban
Rural

$41.0(10.1)$

$78(74.3 \%)$

$27(25.7 \%)$

\section{Education}

No school

$10(9.5 \%)$

Primary school

$38(36.2 \%)$

Secondary school

$42(4.0 \%)$

College school or above

$15(14.3 \%)$

\section{Work status}

Retired

Employed

$7(6.7 \%)$

Unemployed

$10(9.5 \%)$

$88(83.8 \%)$

\section{Children}

No

$36(34.3 \%)$

Yes

$69(65.7 \%)$

$\begin{array}{cc}\text { ASIA grade } & \\ \text { A } & 60(57.1 \%) \\ \text { B } & 15(14.3 \%) \\ \text { C } & 10(9.5 \%) \\ \text { D } & 15(14.3 \%) \\ \text { E } & 5(4.8 \%)\end{array}$

Lesion level

Cervical

$37(35.2 \%)$

Thoracic

$45(42.9 \%)$

Lumbar-sacral

$23(21.9 \%)$

Etiology of SCl

Fall

$9(8.6 \%)$

Impact of object on the body

$12(11.4 \%)$

Car accident

$84(80.0 \%)$

Abbreviations: ASIA, American Spinal Injury Association; SCI, spinal cord injury. 
level. Table 1 presents the demographic and clinical characteristics of the study population.

To assess the impact of age categories on the FSFSI subscales, a oneway analysis of variance with Bonferroni correction was performed. The results of the analysis of variance indicated that older patients suffered from more sexual dysfunction compared with younger patients. Younger patients reported significantly higher scores on the all FSFSI subscales compared with older patients $(P<0.05)$.

Women with SCI reported significantly higher levels of sexual dysfunction compared with healthy women in the control sample (Table 2). Among the FSFSI subscales, lack of vaginal lubrication was reported more frequent in patients with SCI compared with controls. The overall prevalence of FSD based on the cutoff score for the FSFI was $87.6 \%$ compared with only $37 \%$ of healthy controls $(P<0.001)$. Sexual distress (FSDS-R $\geqslant 11$ ) was twice as common in women with SCI compared with controls ( $52 \%$ vs $25 \%, P=0.02$ ). Moreover, women with SCI reported a significantly higher level of sexual distress compared with healthy controls (Table 2). Combined sexual distress or sexual dysfunction was present in $48 \%$ of patients with SCI compared with $21 \%$ of healthy controls $(P<0.01)$.

The scores on the anxiety and depression subscales of the HADS are presented in Table 3. Patients with SCI had significantly more symptoms of anxiety and depression compared with healthy control women. The prevalence of significant anxiety and depression based on cutoff scores was found to be $51 \%$ and $53 \%$, respectively, in women with SCI, compared with $26 \%$ and $29 \%$ in controls $(P=0.015)$.

Correlates of FDS in bivariate analyses $(P<0.1)$ were age, education, living situation, work status, ASIA grade, depression and anxiety. These correlates were entered into the logistic regression model predicting FDS (Table 4). Sexual dysfunction was significantly higher in older patients (odds ratio $(\mathrm{OR})=0.945$ (95\% confidence interval $(\mathrm{CI})=0.897,0.995) P=0.033)$, those with lower education $(\mathrm{OR}=0.164 \quad(95 \% \quad \mathrm{CI}=0.050, \quad 0.139) \quad P=0.003)$, those with complete lesions $(\mathrm{OR}=0.164(95 \% \mathrm{CI}=0.078,0.144) \quad P<0.001)$, those with sexual distress $(\mathrm{OR}=0.920 \quad(95 \% \mathrm{CI}=0.870,0.973)$

Table 2 Sexual functioning scores for each domain in patients with $\mathrm{SCl}$ and controls

\begin{tabular}{lcr}
\hline & Women with SCl $(\mathrm{n}=105)$ & Controls $(\mathrm{n}=1123)$ \\
\hline Desire $^{\mathrm{a}}$ & $2.36(0.70)$ & $3.56(1.80)$ \\
Arousal $^{\mathrm{a}}$ & $2.09(0.64)$ & $3.87(1.13)$ \\
Lubrication $^{\mathrm{a}}$ & $2.56(0.82)$ & $4.40(1.28)$ \\
Orgasma $^{\mathrm{a}}$ & $2.35(1.06)$ & $4.09(1.19)$ \\
Satisfaction $^{\mathrm{a}}$ & $2.78(1.03)$ & $3.75(0.98)$ \\
Pain & $4.45(1.53)$ & $5.01(2.14)$ \\
FSFI total score $^{\mathrm{a}}$ & $17.52(5.93)$ & $23.86(4.43)$ \\
FSDS total score $^{\mathrm{a}}$ & $15.34(5.36)$ & $6.63(2.58)$ \\
\hline Abbreviations: FSDS, Female Sexual Distress Scale; FSFI, Female Sexual Function Index; SCl, \\
spinal cord injury. \\
aStatistically significant at $P<0.10$ according to Benjamini-Hochberg procedure.
\end{tabular}

Table 3 Comparison of the depression and anxiety scores in women with $\mathrm{SCl}$ and controls

\begin{tabular}{lcc}
\hline & Women with SCl $(\mathrm{n}=105)$ & Controls $(\mathrm{n}=1123)$ \\
\hline Depression $^{\mathrm{a}}$ & $8.01(3.49)$ & $4.91(3.20)$ \\
Anxiety $^{\mathrm{a}}$ & $7.93(4.12)$ & $5.28(3.92)$
\end{tabular}

Abbreviation: $\mathrm{SCl}$, spinal cord injury.

a Statistically significant $(P<0.10)$ according to Benjamini-Hochberg procedure.
$P=0.033)$, anxiety $(\mathrm{OR}=0.720(95 \% \mathrm{CI}=0.623,0.832) P<0.001)$ and depression $(\mathrm{OR}=0.669(95 \% \mathrm{CI}=0.549,0.814) P<0.001)$.

\section{DISCUSSION}

Female sexual functioning in women with SCI has received little attention in the research literature. One reason for this is probably because the majority of patients with SCI are male. To our knowledge, only one other study has assessed risk factors for sexual dysfunction among women with SCI. ${ }^{5}$ In the present study, we sought to determine the prevalence of sexual dysfunction and sexual distress and identify risk factors for FSD in patients with SCI. The FSFI has been used in several clinical studies and is a highly sensitive measure for identifying FSD. ${ }^{16}$

Our finding shows that $87 \%$ of women with SCI experience sexual dysfunction is much higher than rates reported among the general population in the Iran, where only $37 \%$ of healthy women without SCI of the same age group experience such problems. ${ }^{13}$ Our findings are consistent with previous reports showing an increased prevalence of sexual dysfunction in women with SCI. ${ }^{5,17}$ We found several specific areas of sexual dysfunction in women with SCI, especially in the areas of sexual desire, arousal, lubrication, orgasm, satisfaction and pain. The prevalence of the FSD in patients with SCI in a previous study (59\%) was substantially lower than in the present study, and likely due to their small sample size $(n=39) .{ }^{17}$

We found that the most significant area of sexual dysfunction in women with SCI was lubrication. A regulated psychogenic sexual arousal and an intact sacral reflex arc are necessary for sexual arousal and vaginal lubrication. Sympathetic cell bodies at the level of T11-L2 have been shown to regulate vaginal blood flow. One study has

\section{Table 4 Factors associated with sexual dysfunction (logistic} regression analysis)

\begin{tabular}{|c|c|c|c|c|c|}
\hline & \multirow[t]{2}{*}{ B } & \multirow[t]{2}{*}{ Odds ratio } & \multicolumn{2}{|c|}{$95 \% \mathrm{Cl}$} & \multirow[t]{2}{*}{ P-value } \\
\hline & & & Lower & Upper & \\
\hline Age & -0.057 & 0.945 & 0.897 & 0.995 & 0.033 \\
\hline \multicolumn{6}{|l|}{ Living situation } \\
\hline Rural & Ref & & & & \\
\hline Urban & 0.127 & 1.13 & -0.130 & 0.202 & 0.823 \\
\hline \multicolumn{6}{|l|}{ Education } \\
\hline None & Ref & & & & \\
\hline Primary school & -1.808 & 0.164 & 0.050 & 0.139 & 0.003 \\
\hline Secondary school & -1.723 & 0.179 & 0.049 & 0.157 & 0.010 \\
\hline College school or above & -1.609 & 0.200 & 0.049 & 0.212 & 0.021 \\
\hline \multicolumn{6}{|l|}{ Work status } \\
\hline Employed & 0.003 & 1.003 & 0.998 & 1.008 & 0.212 \\
\hline Retired & -0.166 & 0.847 & 0.436 & 0.1647 & 0.625 \\
\hline Unemployed & Ref & & & & \\
\hline
\end{tabular}

$\begin{array}{lllllc}\text { ASIA grade } & & & & & \\ \quad \text { Complete (A) } & -1.808 & 0.164 & 0.078 & 0.144 & P<0.001 \\ \quad \text { Incomplete (B,C,D) } & \text { Ref } & & & & \\ \text { FSDS-R } & -0.083 & 0.920 & 0.870 & 0.973 & 0.003 \\ \text { Depression } & -0.329 & 0.720 & 0.623 & 0.832 & P<0.001 \\ \text { Anxiety } & -0.403 & 0.669 & 0.549 & 0.814 & P<0.001\end{array}$

Abbreviations: ASIA, American Spinal Injury Association; Cl, confidence interval; FSDS-R, Female Sexual Distress Scale-Revised; Ref, reference. Nagelkerke $R^{2}=0.404, P<0.001$ 
reported that women with SCI who have preserved T11-L2 pinprick sensation are more able to achieve psychogenetic lubrication. ${ }^{18}$ On the other hand, an intact sacral spinal cord was found to be necessary to achieve reflex lubrication. The findings of the current study are consistent with those of Forsythe and Horsewell who found that decreased vaginal lubrication strongly and negatively affect SCI women's sexual function. ${ }^{7}$ The present study found that pain during intercourse did not significantly affect women's sexual functioning, which is similar to that found in previous studies. ${ }^{4,19}$

Age was another predictor of the FSD in the present study. Others have found that women over age 30 years are associated with a decline in sexual interest. ${ }^{20}$ There are several possible explanations. First, older age is associated with less sexual activity. Second, older age is associated with higher frequency of medical co-morbidities that reduce overall health status and physical vigor. Finally, older women may perceive themselves as less sexually attractive, which may also be influenced by the presence of depression, as our results suggested. Finally, older women may be embarrassed about or ashamed to express their sexuality needs. ${ }^{21}$

The present study extends those observations suggesting that high educational attainment is associated with fewer sexual problems among women with SCI. It is not known why this is the case. However, better-educated women are more likely to have healthier lifestyle that is less physically stressful and emotional demanding. ${ }^{22}$

Another important finding was that depression and anxiety were associated with greater sexual dysfunction. Furthermore, women with SCI experienced more depression and anxiety compared with ageand gender-matched healthy controls. Understandably, women with SCI are more likely to have to cope with the loss of physical functioning after injury, as well as deal with an impaired body image related to their not feeling as sexually attractive. ${ }^{8}$

\section{Limitations}

The present study has several limitations that affect the generalizability of results. First, the patients included in this study were not fully representative of the Iranian women with SCI, but rather involved a convenience sample of women available to be interviewed. Second, given the cross-sectional nature of the study, no conclusions can be drawn regarding the causal nature of the correlates identified in this study. The study also has a number of strengths, including the relatively large sample size compared with previous studies, the use of valid and reliable measures of sexual dysfunction, sexual distress, anxiety and depressive symptoms, and careful neurological grading of the severity of SCI. Future longitudinal studies are needed that assess the incidence and correlates of sexual dysfunction among women with SCI.

\section{CONCLUSION}

We found that sexual dysfunction is highly prevalent among Iranian women with SCI. Sexual dysfunction in these women may be influenced by age, educational attainment, level of anxiety and depressive symptoms, and level and extent of injury. Given the high rate of sexual dysfunction among women following SCI, and the potential role that psychological factors may have in that dysfunction, we recommend that counseling that addresses problems with sexual functioning should be provided to all women immediately following the injury during rehabilitation.

\section{DATA ARCHIVING}

There were no data to deposit.

\section{CONFLICT OF INTEREST}

The authors declare no conflict of interest.

\section{ACKNOWLEDGEMENTS}

We thank all the patients and nurses as well as Iranian Spinal Cord Injury Association who kindly helped us to collect data.

1 Bernhard M, Gries A, Kremer P, Bottiger BW. Spinal cord injury (SCI)-prehospital management. Resuscitation 2005; 66: 127-139.

2 Rahimi-Movaghar V, Saadat S, Rasouli MR, Ganji S, Ghahramani M, Zarei MR et al. Prevalence of spinal cord injury in Tehran, Iran. J Spinal Cord Med 2009; 32: 428-431.

3 Johnson RL, Brooks CA, Whiteneck GG. Cost of traumatic spinal cord injury in a population-based registry. Spinal Cord 1996; 34: 470-480.

4 Anderson KD, Borisoff JF, Johnson RD, Stiens SA, Elliott SL. Spinal cord injury influences psychogenic as well as physical components of female sexual ability. Spinal Cord 2007; 45: 349-359.

5 Celik EC, Akman Y, Kose P, Arioglu P, Karatas M, Erhan B. Sexual problems of women with spinal cord injury in Turkey. Spinal Cord 2014; 52: 313-315.

6 Ferreiro-Velasco ME, Barca-Buyo A, de la Barrera SS, Montoto-Marques A, Vazquez XM, Rodriguez-Sotillo A. Sexual issues in a sample of women with spinal cord injury. Spinal Cord 2005; 43: 51-55.

7 Forsythe E, Horsewell JE. Sexual rehabilitation of women with a spinal cord injury. Spinal Cord 2006; 44: 234-241.

8 Lombardi G, Del Popolo G, Macchiarella A, Mencarini M, Celso M. Sexual rehabilitation in women with spinal cord injury: a critical review of the literature. Spinal Cord 2010; 48: 842-849.

9 Fakhri A, Pakpour AH, Burri A, Morshedi H, Zeidi IM. The Female Sexual Function Index: translation and validation of an Iranian version. J Sex Med 2012; 9: 514-523.

10 Maynard FM Jr, Bracken MB, Creasey G, Ditunno JF Jr, Donovan WH, Ducker TB et al. International standards for neurological and functional classification of spinal cord injury. American Spinal Injury Association. Spinal Cord 1997; 35: 266-274.

11 Wiegel M, Meston C, Rosen R. The female sexual function index (FSFI): cross-validation and development of clinical cutoff scores. J Sex Marital Ther 2005; 31: 1-20.

12 Montazeri A, Vahdaninia M, Ebrahimi M, Jarvandi S. The Hospital Anxiety and Depression Scale (HADS): translation and validation study of the Iranian version. Health Qual Life Outcomes 2003; $1: 14$.

13 Azimi NE, Burri A, Ashrafti F, Fridlund B, Koenig HG, Derogatis LR et al. Psychometric Properties of the Iranian Version of the Female Sexual Distress Scale-Revised in Women. J Sex Med 2014; 11: 995-1004.

14 Benjamini Y, Hochberg Y. Controlling the false discovery rate: a practical and powerful approach to multiple testing. J R Stat SocB 1995; 57: 289-300.

15 O'Brien RM. A caution regarding rules of thumb for variance inflation factors. Qual Quant 2007; 41: 673-690.

16 Rosen R, Brown C, Heiman J, Leiblum S, Meston C, Shabsigh R et al. The Female Sexual Function Index (FSFI): a multidimensional self-report instrument for the assessment of female sexual function. J Sex Marital Ther 2000; 26: 191-208.

17 Lombardi G, Mondaini N, Macchiarella A, Del Popolo G. Female sexual dysfunction and hormonal status in spinal cord injured (SCI) patients. J Androl 2007; 28: 722-726.

18 Sipski ML, Alexander CJ, Rosen RC. Physiologic parameters associated with sexual arousal in women with incomplete spinal cord injuries. Arch Phys Med Rehabil 1997; 78: 305-313.

19 Matzaroglou C, Assimakopoulos K, Panagiotopoulos E, Kasimatis G, Dimakopoulos P, Lambiris E. Sexual function in females with severe cervical spinal cord injuries: a controlled study with the Female Sexual Function Index. Int J Rehabil Res 2005; 28 : 375-377.

20 Sipski ML, Alexander CJ, Rosen RC. Orgasm in women with spinal cord injuries: a laboratory-based assessment. Arch Phys Med Rehabil 1995; 76: 1097-1102.

21 Read J. ABC of sexual health: sexual problems associated with infertility, pregnancy, and ageing. BMJ (Clinical research ed) 1999; 318: 587-589.

22 Pakpour AH, Yekaninejad MS, Zeidi IM, Burri A. Prevalence and risk factors of the female sexual dysfunction in a sample of infertile Iranian women. Arch Gynecol Obstetr 2012; 286: 1589-1596. 